\title{
Periodontal Disease: Causes, Symptoms and Treatment
}

ISSN: 2637-7764

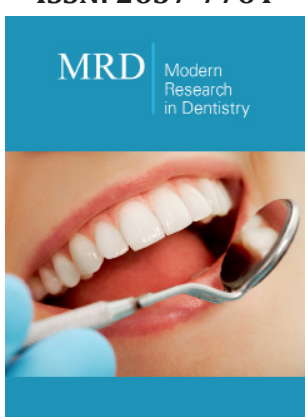

*Corresponding author: Baburoa $\mathrm{N}$ Chandakavathe, DSTS Mandal's College of pharmacy, Solapur, Maharashtra, India

Submission: 海 June 03, 2019

Published: 㘹June 07, 2019

Volume 4 - Issue 2

How to cite this article: Baburoa $\mathrm{N}$ Chandakavathe. Periodontal Disease: Causes, Symptoms and Treatment. Mod Res Dent. 4(2). MRD.000582.2019. DOI: 10.31031/MRD.2019.04.000582

Copyright@ Baburoa N Chandakavathe, This article is distributed under the terms of the Creative Commons Attribution 4.0 International License, which permits unrestricted use and redistribution provided that the original author and source are credited.

\author{
Baburoa N Chandakavathe* \\ DSTS Mandal's College of pharmacy, India
}

\section{Opinion}

In the present scenario "periodontitis" is major dental disorder among all the dental diseases, it is also called as gum disease, periodontitis is characterized by annihilation of connective tissue and dental bone support after an inflammatory host response secondary to infection by bacteria. In its premature stage, called gingivitis, the gums become enlarged, red, and may bleed. Periodontal diseases are of several types, all of which begin with an infection of the gums that can proliferate into the bones and ligaments of the teeth. In the beginning, this disease can be detected by a dentist during regular check-ups. It is the major cause for tooth loss in children and adults. If it is not treated bone and gums seriously damaged, the teeth may fall out or has to be removed. More than $50 \%$ of adults are suffering from some form of periodontal disease and even younger population also predominantly affected [1].

\section{Stages periodontal disease}

Different stages of periodontal disease so far investigated briefly explained here; the earliest stage called gingivitis, during this stage the gums begin to swell, puffy and get red may even bleed during flossing but bone remains healthy in this stage. If it is not noticed and diagnosed leads to next stage called periodontitis, in this stage the gums begin to separate from the teeth, the underlying bone is damaged, pockets form and, the gums recede [1]. plaque containing bacteria spreads into the pockets, it becomes difficult to keep tooth clean and to control the disease progress. In the final stages of the disease, the pockets continue to get deeper and further destruction of bone. Moreover, the bacteria that live in these pockets are more virulent and put in even further for the progress of the disease. If not treated, ultimately teeth will fall out and/or loosen.

\section{Causes of periodontal disease}

Major cause of periodontitis is the microorganisms present in the food plaque release toxins after metabolic reactions that irritate and inflammation of gums [2]. If plaque not removed from teeth, it becomes hard, spongy like substance known as calculus. If calculus is formed on roots of teeth just below the gums, it irritates the gums and contributes to even more accumulation of plaque and progress of disease [3]. Apart from this major cause there are infinite numbers of risk factors also contribute for periodontitis such as chewing tobacco or smoking, Poor oral hygiene, Hormonal changes in girls/women, poorly fitting bridges, genetic susceptibility, poor teeth alignment, medications, defective fillings, pregnancy, systemic diseases such as AIDS or diabetes etc $[2,3]$.

\section{Symptoms of periodontal disease}

Early symptoms of this disease can be detected by a dentist, as the disease progresses other symptoms start to appear. Symptoms such as bleeding of gums during brushing, swollen or red gums, loosened teeth, continuous bad breath, receded gums, changes in the way teeth fit together when eating etc [4].

\section{Treatment of periodontal disease}

If this disease is diagnosed in early stage of gingivitis, it can be treated with a thorough cleaning by dentist or oral hygienist. If the disease has progressed beyond gingivitis, the 
treatment may involve a process called "deep cleaning" or "root planning," this involves cleaning and smoothing of root surfaces of the teeth to remove calculus and bacterial deposits below the gum line so that the gums can heal around them $[4,5]$. Depending on how deep the disease has progressed, dentist or periodontist may still advice surgical treatment. The long-term studies are needed to find out if using medications reduces the need for surgery and whether they are effective over a long period of time. Some medications that are currently used are

a) prescription mouth rinse containing chlorhexidine,

b) small piece of gelatine filled with chlorhexidine,

c) gel that contains an antibiotic called doxycycline,

d) tiny round particles that contain the minocycline antibiotic, and

e) low dose of the medication doxycycline that keeps destructive enzymes in check.

Apart from above mentioned chemotherapy some surgical procedures are also suggested if need arises such as flap surgery, bone surgery, and bone-tissue grafts surgery.

\section{Prevention of periodontal disease}

The more popular saying quote "prevention is better than cure" so the best way to prevent periodontal disease is to practice good oral hygiene. Brushing every day, taking a balanced diet and regular visits to oral hygienist are essential to keep periodontal disease away. And if you do so, you'll increase your chances of maintaining your teeth for a lifetime $[4,6]$.

\section{References}

1. Periodontal (Gum) Disease. Causes, Symptoms, and Treatments. US Department of Health and Human Services, National Institutes of Health, Bethesda, Maryland, USA.

2. Gum Disease. A Guide to Periodontal Disease. California Dental Association, USA.

3. James P, Worthington HV, Parnell C, Harding M, Lamont T, et al. (2017) Chlorhexidine mouth rinse as an adjunctive treatment for gingival health. Cochrane Database Syst Rev 3: CD008676.

4. (2005) Treating periodontal diseases. Journal of the American Dental Association 136: 127.

5. (2015) Periodontal (Gum) Disease: Causes, Symptoms and Treatments, National Institute of Dental and Craniofacial Research. National Institutes of Health, Bethesda, Maryland, USA.

6. (2015) Academy of General Dentistry. What is gum disease? 